\title{
A CHARACTERISTIC PLANETARY FEATURE IN CAUSTIC-CROSSING HIGH-MAGNIFICATION MICROLENSING EVENTS
}

\author{
Doeon Kim and Cheongho Han \\ Program of Brain Korea 21, Department of Physics, Chungbuk National University, \\ Cheongju 361-763, Republic of Korea \\ E-mail:dekim,cheongho@astroph.chungbuk.ac.kr \\ (Received April 18, 2009; Accepted May 8, 2009)
}

\begin{abstract}
We propose a diagnostic that can resolve the planet/binary degeneracy of central perturbations in caustic-crossing high-magnification microlensing events. The diagnostic is based on the difference in the morphology of perturbation inside the central caustics induced by a planet and a wide-separation binary companion. We find that the contours of excess exhibit a concentric circular pattern around the caustic center for the binary-lensing case, while the contours are elongated or off-centered for the planetary case. This difference results in the distinctive features of the individual lens populations in the residual of the trough region between the two peaks of the caustic crossings, where the shape of the residual is symmetric for binary lensing while it tends to be asymmetric for planetary lensing. We determine the ranges of the planetary parameters for which the proposed diagnostic can be used. The diagnostic is complementary to previously proposed diagnostics in the sense that it is applicable to caustic-crossing events with small finite-source effect.
\end{abstract}

Key words : gravitational lensing - planets and satellites: general

\section{INTRODUCTION}

Since the first detection in 2004, the number of extrasolar planets discovered by the microlensing method is rapidly increasing (Bond et al. 2004; Udalski et al. 2005; Beaulieu et al. 2006; Gould et al. 2006; Gaudi et al. 2008; Dong et al. 2008; Bennett et al. 2008). The microlensing method is important because it is sensitive to planets that are generally inaccessible to other methods, in particular cool planets at or beyond the snow-line, very low-mass planets, planets orbiting lowmass stars, free-floating planets, and even planets in external galaxies.

The microlensing signal of a planet is a shortduration perturbation to the smooth standard light curve of the primary-induced lensing event. The duration of the perturbation is several days for a gas giant and several hours for an Earth-mass planet. The typical cadence of the current lensing surveys is roughly a day and thus planets cannot be detected by the survey observations alone.* ${ }^{*}$ To achieve the cadence required to detect planets, current planetary lensing

Corresponding Author: C. Han

* To dramatically increase the monitoring frequency enough to detect planets with masses down to below the Earth mass, it is planned to build a network of telescopes located in the southern hemisphere for exclusive use for microlensing planet search (Korea Microlensing Telescope Network). Aiming to be fully operational in 2013, the network will be composed of $\sim 2-3$ telescopes with an aperture of each telescope of $\sim 1.5 \mathrm{~m}-2 \mathrm{~m}$ equipped with a very wide-field $\left(\sim 2^{\circ} \times 2^{\circ}\right)$ camera. searches are being operated by using a combination of survey and follow-up observations, where alerts of ongoing events are issued by the survey observations (OGLE: Udalski et al. 2008; MOA: Bond et al. 2002) and the alerted events are intensively monitored by the follow-up observations (Micro-FUN: Dong et al. 2006; PLANET: Kubas et al. 2008). However, the number of telescopes available for follow-up observations is much less than what is needed for intensive monitoring of all alerted events and thus observations are focused on events which will yield high probability of planet detections. Currently, the highest priority is given to highmagnification events because (1) these events intrinsically have high planet detection efficiency due to the passage of the source trajectory close to the region of central perturbations, (2) follow-up observations can be prepared in advance due to the predictable time of the perturbation near the peak of lensing light curve, and (3) much more numerous small-aperture telescopes can be used for follow-up observations due to the greatly enhanced source star flux around the time of perturbation.

Although high-magnification events have an important merit of yielding high sensitivity to planets, they also have a shortcoming of the difficulty in the interpretation of observed signals due to several possible degeneracies. One of these degeneracy is caused by the fact that a central perturbation can be produced not only by a planet but also by a very wide or very close binary with roughly equal mass components. Fortunately, the perturbation patterns produced by a planetary and a 
binary companion are intrinsically different and thus it is in principle possible to discriminate between the planetary and the binary interpretations. However, this discrimination usually requires detailed modelling of the light curve which demands time-consuming search for a solution of the lens parameters in the vast space of many parameters. Therefore, a simple diagnostic that can resolve the planet/binary degeneracy will be very useful not only for the immediate identification of the nature of the perturbation but also for the preparation of observational strategies for better characterization of planetary systems.

Several diagnostics have been proposed to resolve the planet/binary degeneracy. Han \& Gaudi (2008) proposed a diagnostic that is applicable to planetary perturbations with a double-peak structure occurring during the passage of the source trajectory close to the two strong cusps of a central caustic. They showed that the shape of the intra-peak region of a planetary event is different from that of a binary event due to the small weak cusp of the planetary central caustic located between the two strong cusps. Han (2009b) proposed another diagnostic that can be applicable to high-magnification events where the angular extent of the perturbation region is significantly smaller than the size of the source star and thus finite-source effect is very severe. He showed that for binary lensing there always exist two well-developed dips in the residual of the light curve from that of a single-lensing event at the moments of the caustic center's entrance into and exit from the source star surface, while there can be no dip at both or either of the moments for planetary lensing. In this paper, we propose another diagnostic that is applicable to caustic-crossing high-magnification events with well resolve trough region between the two peaks of the caustic crossings.

The paper is organized as follows. In $\S 2$, we briefly describe the characteristics of the central caustics produced by a planet and a binary companion. In $\S 3$, we compare the morphology of the perturbations induced by the two different populations of companions, paying special attention to the morphology inside the central caustics. We then describe how the diagnostic is used and why the use of the diagnostic is possible. In $\S 4$, we discuss the range of planetary parameters for which the proposed diagnostic can be applied. In $\S 5$, we summarize the result and conclude.

\section{PLANETARY AND BINARY CENTRAL CAUSTICS}

For planetary lensing, the central caustic refers to a small caustic located close to the primary lens. The caustic forms a closed figure that is composed of concave curves that meet at four cusps. The size of the planetary central caustic as measured by the separation between the two cusps located on the primary-planet axis is related to the planetary lensing parameters by
Chung et al. (2005)

$$
\Delta \xi_{p} \sim \frac{4 q}{\left(s-s^{-1}\right)^{2}}
$$

where $s$ represents the projected primary-planet separation normalized by the Einstein radius corresponding to the total mass of the lens system and $q$ is the planet/primary mass ratio. Then, the caustic size is directly proportional to the planet/primary mass ratio. In the limiting cases of a very wide and a very close planets, the caustic size depends on the separation by

$$
\Delta \xi_{p} \propto \begin{cases}s^{-2} & \text { for } s \gg 1, \\ s^{2} & \text { for } s \ll 1 .\end{cases}
$$

While the size of the central caustic depends on both the mass ratio and the separation, its shape depends solely on the separation. When the shape is quantified as the ratio of the vertical width $\Delta \eta_{p}$ to the horizontal width $\Delta \xi_{p}$, the width ratio is related to the planetary separation by

$$
\frac{\Delta \eta_{p}}{\Delta \xi_{p}} \sim \frac{\left(s-s^{-1}\right)\left|\sin ^{3} \phi\right|}{\left(s+s^{-1}-2 \cos \phi\right)^{2}},
$$

where $\cos \phi=(3 / 4)\left(s+s^{-1}\right)\left\{1-\left[(32 / 9)\left(s+s^{-1}\right)^{2}\right]^{1 / 2}\right\}$. For the range of planetary separations where the size of the central caustic, which is directly proportional to the planet-detection efficiency, is not negligible (Han 2009a), the width ratio of the planetary central caustic is substantially smaller than unity, implying that the caustic is elongated along the primary-planet axis. In addition, three of the four cusps of the caustic lean toward the primary direction and thus the caustic has an arrowhead shape where the sharp point directs toward the planet. For a given mass ratio, a pair of central caustics with separations $s$ and $s^{-1}$ are identical to the first order of approximation.

When the separation between the components of a binary is very wide $(s \gg 1)$, two sets of caustics are formed around the individual lens components. Each of the caustics has four cusps among which two are located on the binary axis and the others are located off the axis. We refer the binary central caustic as the one located close to the lens component close to which the source trajectory approaches. The size of the binary central caustic as measured by the way similar to the planetary case is

$$
\Delta \xi_{b} \sim \frac{4 \gamma}{(1-\gamma)^{1 / 2}} ; \quad \gamma=\frac{q}{(1+q) s^{2}},
$$

where $\gamma$ is the shear exerted by the companion. The width ratio of the binary central caustic defined also by the way similar to the planetary case is (Han et al. 2005)

$$
\frac{\Delta \eta_{b}}{\Delta \xi_{b}} \sim\left(\frac{1-\gamma}{1+\gamma}\right)^{1 / 2}
$$



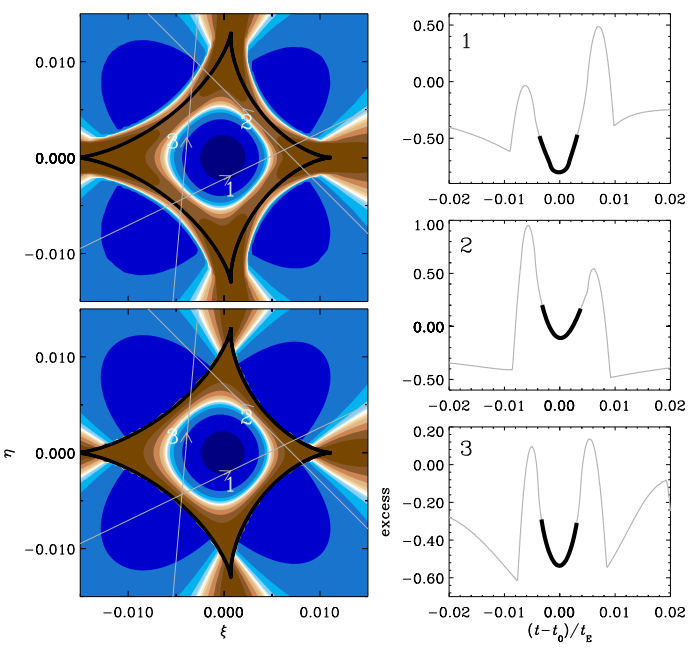

Fig. 1.- Color-scale maps of magnification excess around the central caustic induced by a wide-separation binary companion. The coordinates $(\xi, \eta)$ are centered at the caustic center, the abscissa is parallel with the axis connecting the two lens components, and lengths are normalized by the Einstein radius corresponding to the total mass of the lens system. The companion is on the left. Colors are chosen such that the regions with brown and blue-tone colors represent the areas where the magnification is higher $(\epsilon>0)$ and lower $(\epsilon<0)$ than the single-lensing magnification without the companion, respectively. For each tone, the color scale becomes darker at the excess levels of $|\epsilon|=2 \%, 4 \%, 8 \%, 16 \%, 32 \%$, and $64 \%$, respectively. The map in the upper left panel is constructed by considering the finite-source effect while the map in the lower left panel is for a point source. The straight lines with arrows represent the source trajectories of caustic-crossing events where the residuals of the light curves of the resulting events from that of a single-lensing event are presented in the right panels marked by the corresponding numbers. The normalized separation and the mass ratio between the lens components are $(s, q)=(7.0,0.7)$. The time of the residual curve is measured with respect to the time of the closest approach of the source trajectory to the center of the caustic, $t_{0}$, and it is normalized by the Einstein time scale $t_{\mathrm{E}}$.

For a wide-separation binary, the shear is very small because $\gamma \propto s^{-2}$. Then, the width ratio becomes $\eta_{b} / \xi_{b} \rightarrow 1$, implying that the horizontal and vertical width are nearly identical for the central caustic of a wide-separation binary. In addition, the shape of the caustic is symmetric with respect to both the binary axis and the axis normal to the binary axis and thus the caustic has a shape of a hypo-cycloid with four cusps. The caustic induced by a close binary is similar to the caustic of the wide-separation binary with a separation of $s^{-1}$ both in size and shape.
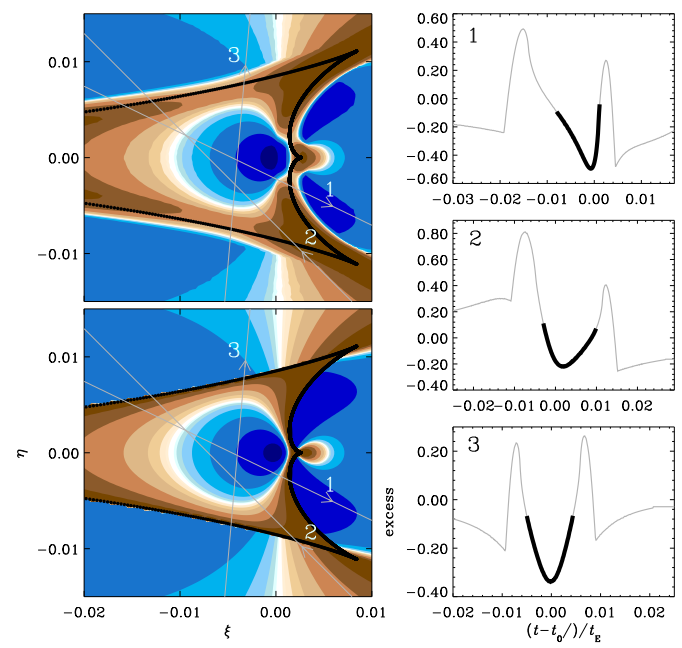

Fig. 2.- Color-scale maps of magnification excess around the central caustic induced by a planet. Notations are same in Fig. 1. The normalized separation and the mass ratio between the lens components are $(s, q)=\left(1.3,5 \times 10^{-3}\right)$.

\section{PERTURBATION PATTERN INSIDE CAUSTIC}

To see the difference between the morphology of the central perturbations induced by a planet and a binary companion, we produce excess maps in the central regions of representative lens systems of the individual lens populations. The magnification excess represents the fractional deviation of the lensing magnification $A$ from the single-lensing magnification without the companion $A_{0}$, i.e.,

$$
\epsilon=\frac{A-A_{0}}{A_{0}} .
$$

In Figure 1 and 2, we present the excess maps for a binary and a planetary lens systems, respectively. The maps are represented in color scale. In each map, the regions with brown and blue-tone colors represent the areas where the magnification is higher $(\epsilon>0)$ and lower $(\epsilon<0)$ than the single-lensing magnification, respectively. For each tone, the color scale becomes darker at the excess levels of $|\epsilon|=1 \%, 2 \%, 4 \%, 8 \%$, $16 \%, 32 \%$, and $64 \%$, respectively. The map on the lower left panel is for a point-source case, while the map in the upper left panel is constructed considering the finite size of the source star. We assume that the source radius normalized by the Einstein radius is $\rho=0.002$, which roughly corresponds to the case of an event produced by a low-mass stellar lens with a mass $M \sim 0.3 M_{\odot}$ located at $6 \mathrm{kpc}$ occurred on a background source star with a radius equivalent to that of the Sun and located toward the Galactic bulge field at a distance of $8 \mathrm{kpc}$. The coordinates $(\xi, \eta)$ are centered at the center of the caustic that is located at the position with an offset from the position of the primary 
of

$$
\boldsymbol{\delta}= \begin{cases}\mathbf{s}^{-1} q /(1+1) & \text { for } s>1, \\ -\mathbf{s}\left[(1+q)^{-1}-1\right] & \text { for } s<1,\end{cases}
$$

where the sign is such that it is positive when the offset vector is directed toward the companion and vise versa. All lengths are normalized by the Einstein radius corresponding to the total mass of the lens system. Also presented in the right panels of each figure are the residuals of the light curves of several caustic-crossing events resulting from the source trajectories marked on the map with corresponding numbers. The separations and the mass ratios between the lens components are $(s, q)=(7.0,0.7)$ for the binary and $\left(1.3,5 \times 10^{-3}\right)$ for the planetary lens systems.

From the comparison of the excess maps, we find the following tendencies of the perturbation patterns inside the caustic. The general pattern of excess inside the caustic is that contours of excess roughly follow the caustic line in the outer region close to the caustic, but contours become more circular as the distance from the caustic increases. For binary lensing, the contours exhibit a concentric circular pattern around the caustic center and this concentric circular region occupies an important portion of the area inside the caustic. For planetary lensing, on the other hand, the circular contours appear only in a small localized region. In addition, the contours are off-centered even in the circular-contour region.

The difference in the morphology of the perturbations results in distinctive features of the individual lens populations in the residual of the trough region between the peaks of the caustic crossings. The shape of the residual curve is symmetric for binary lensing, while it tends to be asymmetric for planetary lensing, as shown in the right panels of Figure 1 and 2. Therefore, the shape of the residual curve in the intra-peak trough region can be used to distinguish between the planetary and binary interpretations.

However, we note that although the general shape of the residual curves of planetary events is asymmetric, it can be symmetric for some cases. Such a case happens for an event produced by a source trajectory with an orientation angle with respect to the primaryplanet axis is close to $90^{\circ}$, e.g., the trajectory marked by " 3 " in the maps of Figure 2. This symmetry happens because the perturbation pattern is symmetric with respect to the primary-planet axis although asymmetric with respect to the axis perpendicular to the axis. This implies that symmetry of the residual curve does not necessarily confirm that the perturbation is caused by a binary companion, although asymmetry of the residual curve confirms that the perturbation is produced by a planet.

\section{DISCUSSION}

The proposed diagnostic can be used for causticcrossing high-magnification events where the trough re-

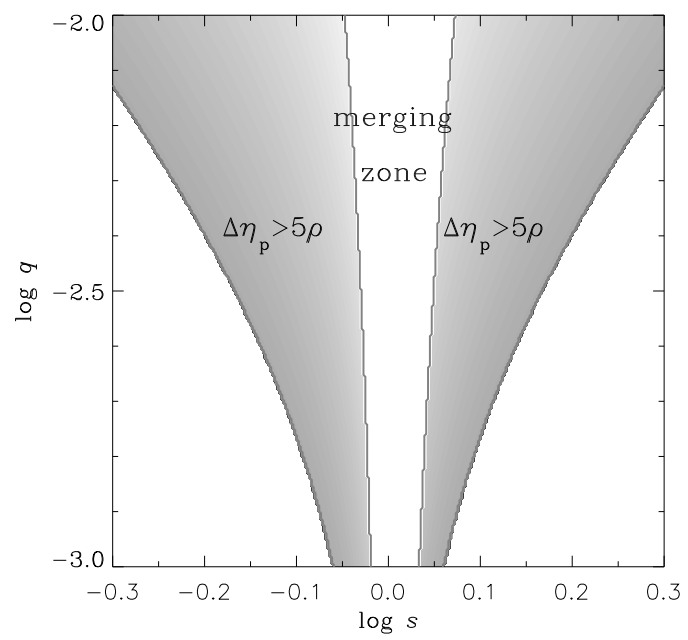

Fig. 3. - The range in the planetary parameter space where the proposed diagnostic can be applicable for the resolution of the planet/binary degeneracy (shaded region). The merging zone represents the area where the central and the planetary caustics merge together, forming a single set of caustics.

gion between the two peaks at the caustic crossings is well resolved. This implies that resolving the trough region is very important to discriminate between the two interpretations. However, even if the region is well resolved, it would be difficult to use the diagnostic if the intra-peak region is not well developed. Such a case occurs when the angular size of the source star is substantial compared to the size of the caustic. In this case, the finite-source effect is severe and thus the perturbation pattern inside the caustic is seriously washed out.

We determine the ranges of planetary parameters for which the proposed diagnostic can be applied by setting a criterion such that the size of the central caustic is substantially greater than the source size. Figure 3 shows the range determined based on this criterion. We assume that the vertical width of the caustic $\Delta \eta_{p}$, which is short side of the caustic, should be 5 times greater than the normalized radius of a typical source radius of $\rho=0.002$. As the planetary separation approaches the Einstein radius $(s \rightarrow 1)$, not only the central caustic but also the planetary caustic, which is the other caustic located away form the primary lens, become bigger and eventually the two caustics merge together. In this case, the shape of the central caustic and the resulting perturbation are greatly changed and thus the proposed diagnostic cannot be applied. We therefore exclude the region of caustic merger. From the figure, it is found that the proposed diagnostic will be useful for events produced by planetary systems with massive planets.

The proposed diagnostic is complementary to the other diagnostics proposed before. It is complementary 
to the diagnostic proposed by Han \& Gaudi (2008) in the sense that the diagnostic is applicable to causticcrossing events while that of Han \& Gaudi (2008) is applicable to non-caustic-crossing events. It is complementary to the diagnostic proposed by Han (2009b) in the sense that the diagnostic is applicable to events with small finite-source effect while the diagnostic of Han (2009b) is applicable to events with severe finitesource effect.

\section{CONCLUSION}

In this paper, we propose a diagnostic that can be used to resolve the planet/binary degeneracy of central perturbations in caustic-crossing high-magnification microlensing events. The diagnostic is based on the difference in the morphology of perturbation inside the central caustics induced by a planet and a wide-separation binary companion. We found that the contours of excess exhibit a concentric circular pattern around the caustic center for the binary-lensing case, while the contours are elongated or off-centered for the planetary case. This difference results in the distinctive features of the individual lens populations in the residual of the trough region between the two peaks of the caustic crossings, where the shape of the residual is symmetric for binary lensing while it tends to be asymmetric for planetary lensing. We determined the range of the planetary parameters for which the proposed diagnostic can be used and found that the diagnostic would be useful for events produced by planetary systems with massive planets. Combined with the diagnostics proposed before, therefore, the planet/binary degeneracy can be resolved for many cases of central perturbations.

\section{ACKNOWLEDGEMENTS}

This work was supported by the research grant of the Chungbuk National University in 2007.

\section{REFERENCES}

Beaulieu, J.-P., et al., 2006, Discovery of a cool planet of 5.5 Earth masses through gravitational microlensing, Nature, 439, 437

Bennett, D. P., et al., 2008, A Low-Mass Planet with a Possible Sub-Stellar-Mass Host in Microlensing Event MOA-2007-BLG-192, ApJ, 684, 663

Bond, I. A., et al., 2002, Study by MOA of extrasolar planets in gravitational microlensing events of high magnification, MNRAS, 333, 71

Bond, I. A., et al., 2004, OGLE 2003-BLG-235/MOA 2003-BLG-53: A Planetary Microlensing Event, ApJ, 606, L155

Chung, S., et al., 2005, Properties of Central Caustics in Planetary Microlensing, ApJ, 630, 535

Dong, S., et al., 2006, Planetary Detection Efficiency of the Magnification 3000 Microlensing Event OGLE2004-BLG-343, ApJ, 642, 842
Dong, S., et al., 2008, Microlensing Event MOA2007-BLG-400: Exhuming the Buried Signature of a Cool, Jovian-Mass Planet, ApJ, submitted (arXiv:0809.2997)

Gaudi, B. S., et al., 2008, Discovery of a Jupiter/Saturn Analog with Gravitational Microlensing, Science, 319,927

Gould, A., et al., 2006, Microlens OGLE-2005-BLG-169 Implies That Cool Neptune-like Planets Are Common, ApJ, 644, L37

Han, C., 2009a, Distinguishing Between Planetary and Binary Interpretations of Microlensing Central Perturbations Under the Severe Finite-Source Effect, ApJ, 691, 9

Han, C., 2009b, Microlensing Zone of Planets Detectable Through the Channel of High-Magnification Events, ApJ, 691, 452

Han, C. \& Gaudi, B. S., 2008, A Characteristic Planetary Feature in Double-Peaked, High-Magnification Microlensing Events, ApJ, 689, 53

Han, C., Gaudi, B. S., An, J. H., \& Gould, A., 2005, Microlensing Detection and Characterization of WideSeparation Planets, ApJ, 618, 962

Kubas, D., et al., 2008, Limits on additional planetary companions to OGLE 2005-BLG-390L, A\&A, 483, 317

Udalski, A., et al., 2005, INTEGRAL IGR J18135-1751 = HESS J1813-178: A New Cosmic High-Energy Accelerator from keV to TeV Energies, ApJ, 629, L109

Udalski, A., et al., 2008, The Optical Gravitational Lensing Experiment. Final Reductions of the OGLEIII Data, Acta Astronomica, 58, 69 